\title{
Implant Associated Local Recurrence in Primary Bone Sarcoma
}

\author{
ZSOMBOR PÁNTI',2, BOGDAN CRETU1 ${ }^{1}$, CORINA PANAITESCU ${ }^{1}$, MIHAI NICA ${ }^{1,2 *}$, CAMELIA TECU ${ }^{3}$, \\ AUGUSTIN SEMENESCU ${ }^{3}$, DRAGOS ENE ${ }^{4,2}$, RAZVAN ENE $^{1,2}$ \\ ${ }^{1}$ Emergency University Hospital of Bucharest, Orthopaedic and Traumatology Department, 169 Splaiul Independentei, \\ 050098, Bucharest, Romania \\ ${ }^{2}$ University of Medicine and Pharmacy "Carol Davila" Bucharest, 8 Eroii Sanitari Str., 050474, Bucharest, Romania \\ ${ }^{3}$ University Politehnica of Bucharest, 1-3 Splaiul Independentei, 060042, Bucharest, Romania \\ ${ }^{4}$ Emergeny Clinical Hospital of Bucharest, Surgery Department, 8 Calea Floreasca, 014461, Bucharest, Romania
}

\begin{abstract}
Hydroxyapatite (HA) covered implants are widely used in orthopedic reconstructive surgery. Their biological benefits were highlighted by several studies in the past decades in which the most important ones are the osteoconductive and osteoinductive capacity, which enhances osteoblast adherence, proliferation and bone extracellular matrix formation. In bone sarcomas the microenvironmental changes are crucial for tumor growth, new bone formation especially in osteoblastic osteosarcomas (OS). Nowadays limb sparing surgery and maintaining the quality of patient's life are the main two goals in bone sarcoma treatment. The survival rate of patients with OS has been significantly improved in the past 3-4 decades due to the advanced chemotherapy protocols. The prognosis still depends on the early diagnosis and the histological type of the sarcoma. Tumor removal and reconstruction is a crucial moment for prognosis, studies showed that high grade sarcomas have a greater potential for local recurrence and early distant metastases. Metastasis can occur in early stages, even if there are not detectable at the time of surgery. Furthermore, the micro-environmental changes can facilitate sarcoma cell proliferation even if the tumor resection was performed in macroscopically normal bone tissue. We present a case of osteosarcoma in which local recurrence has occurred despite of chemotherapy and wide resection of the tumor. We can ask the following question can an implant increases the risk of local recurrence in sarcoma surgery? The aim of the study was to understand the underlying molecular pathway for sarcoma cell proliferation in HA covered implants.
\end{abstract}

Keywords: Hydroxyapatite, osteosarcoma, local recurrence

Limb salvage surgery and reconstruction in primary bone malignancies has become the main focus in tumor surgery. Comparing the data from the early 80 's, the number of amputations has significantly decreased in the last decades and due to the new chemotherapeutical therapies the survival rate of patients with primary bone tumors has significantly improved [1]. Nowadays the increased number of limb sparing and reconstructive surgery led to a high number of complications like infections, implant loosening, and local recurrence. Local recurrence dramatically worsens the prognostics and the survival rate.

Choosing the right surgical approach for biopsy and establishing the safe zones of a resection site, can improve the outcome[2-4]. Any patient with a suspected bone tumor pathology should be discussed in a multidisciplinary team, from the beginning, to carefully plan the approach for biopsy, and to determine the most suggestive zone of the tumor tissue.[5] The role of immunohistochemistry (IHC) exam showed its prognostic role in several recent studies. Due to the help of these IHC markers, a potential resistance to chemotherapy can be predicted thus a greater chance to local recurrence [6,7].

Any changes of the microenvironment of the tumor cell, can lead to faster tumor cell proliferation. An inflammation process after biopsy can lead to an increased cell proliferation by the change of the local environment. The elevated growth factors in this inflammation zone acts on tumor cells and lead to further tumor growth [8].

The chemotherapy has the major role of controlling this fast growth phase after biopsy and controlling the micro metastases and local recurrence [9].

Hydroxyapatitate (HA) implants are widely used in reconstruction surgery, with an important osteoconductive and osteoinductive role, just like many other implants used in osteoconductive and osteoinductive reconstructive surgery [10]. Cell adhesion on these HA covered surfaces is the key response for the integration of the implants. Bone ongrowth on these surfaces is crucial for a secondary biological fixation of the implant. These properties of the HA being present in a normal bone extracellular matrix, can help osteosarcoma cells for further proliferation, and can lead to local recurrence $[11,12]$. The use of these HA covered implants and their relationship to local recurrence in sarcoma surgery were discussed in a reduced number of studies, the underlying molecular pathway is not well understood. 
The aim of this paper is to highlight the importance of local sarcoma recurrence in limb sparing surgery despite of chemotherapy and en-bloc resection of the tumor. The following question can be asked: can an implant increase the risk of local recurrence in sarcoma surgery?

\section{Experimental part}

Materials and methods

We present a case of a 21-year-old female patient, who presented to our Orthopedic department at the University Emergency Hospital of Bucharest, with pain of the distal region of the right thigh in 2016. The primary x-ray scan showed a lytic bone lesion with a marked periosteal reaction. Whole body scintigraphy described an increased metabolic activity and high vascularization of the distal metaphyseal region of the femur. The vascularization of the tumor showed a chaotic venous circulation seen on the angiography (Fig. 1.) At this time no pulmonary or other bony determination could be seen on the CT scans.

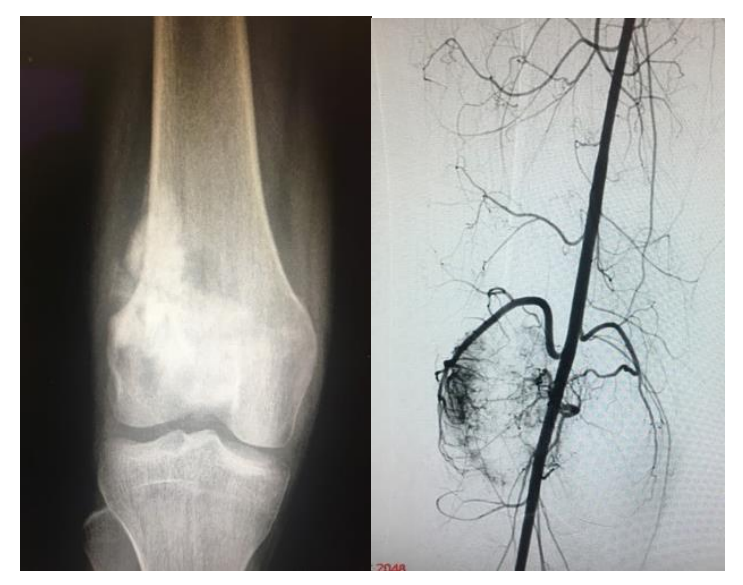

\author{
Fig.1.Showing an osteosclerotic \\ bone forming tumor with elevated \\ periosteum (Codman sign) a highly \\ vascularized tumor lesion at the \\ distal region of the femur
}

The biopsy of the distal femur was performed in an anterior approach taken in consideration that the tumor was easier to approach without harming a healthy bone cortex. The histological examination revealed the presence of an osteoblastic osteosarcoma, with an elevated 30\% KI67 proliferation index (Fig. 2).

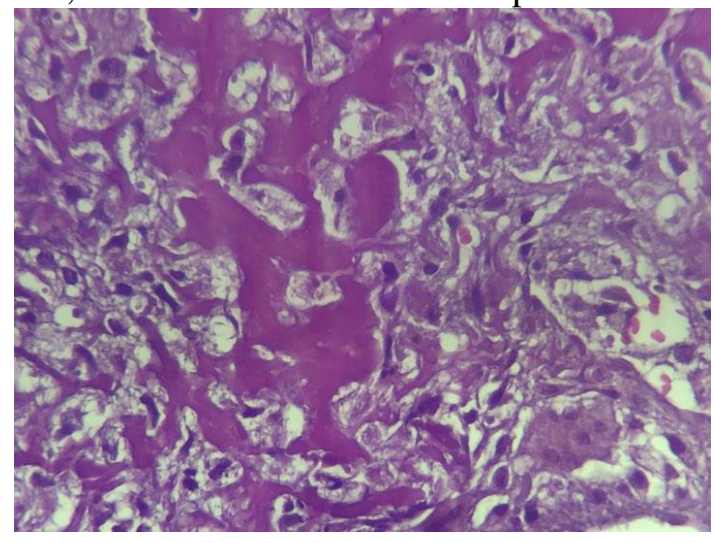

Fig. 2. (Right)HematoxilineEosine histological examination, showing multiple atypical nuclear cells and osteoid presence. The Ki67 proliferation index, was over $30 \%$ of the examined field on the IHC exam

\section{Results and discussions}

Chemotherapy was initiated for 6 weeks, followed by wide resection duo to Enneking IIB classification and reconstruction with a modular implant for the distal femur. The functional results after surgery were good, full weight bearing was achieved in the third day.

The anatomical and histological response to the chemotherapy was not satisfactory, the necrotic index regarding to Huvossystem was grade 1 with little evidence of necrosis. Chemotherapy was continued after reconstruction surgery at the oncology department.

X-ray and CT scan follow-up in 6 to 6 months after resection surgery was good for the first 2 years. In the beginning of 2018 an $\mathrm{x}$-ray scan reveals the presence of a new-bone formation at the anterolateral part of the distal femoral implant. The x-ray also revealed a bone formation of the proximal bone-implant interface as good a sign for secondary bone fixation of the implant (Fig. 3.). 

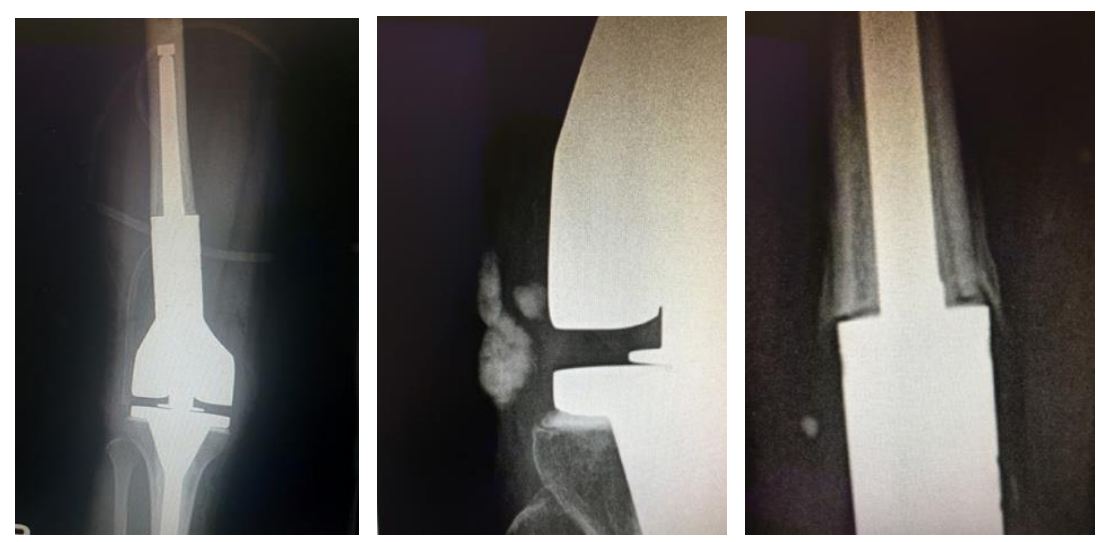

Fig. 3. From the right the postoperative $\mathrm{X}$-ray of the resection and reconstruction, after 1 year a sclerotic tissue can be seen near the joint line and bridging at the level of the implant and bone junction where a small sclerotic lesion can also be evidenced.

Wide resection of the bone island was performed, with the result of a local recurrence of osteobalstic osteosarcoma. A novel chemotherapy was applied with a bad response till the beginning of 2019 when a new x-ray, and PET scan confirms a major local recurrence of the tumor at the level of the bone-implant interface (Fig. 4.)
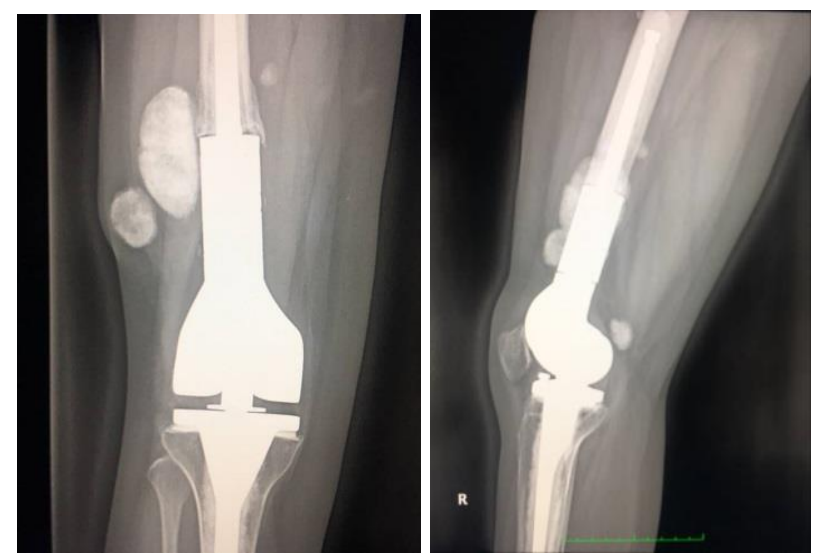

Fig. 4. Bone forming tumor tissue can be seen after 2 years of resection and reconstruction, with repeated chemotherapy

Pulmonary metastases were not detected at this time. Amputation of the lower limb was performed at the level of the proximal thigh, followed by another histology and IHC exam. There was no evidence of post chemotherapy necrosis in the tumor tissue. And there were no signs of phenotypic changes of the tumor histology subtype.

This case underlines the potential risk of local recurrence in sarcoma surgery using HA cover implants despite of clear or cancer free resection sites. As previous studies showed, the most common genetic alteration in malignant tumors is the loss of function of the p53 gene located on chromosome 17 [13]. The role of this gene was demonstrated in several studies playing an important role in cell cycle arrest or apoptosis. Another common possibility for increased cell proliferation is the increased level of MDM2 protein which inhibits the apoptotic function of the p53 gene [14]. These molecular mechanisms can lead to increased cell proliferation, which enhances further genetic alteration in the cell thus leading to numerous chromosomal alterations.

Taken in consideration these common genetic aspects of an osteoblastic osteosarcoma and the osteoinductive and osteoconductive role of the HA, osteoblast proliferation with the underlying genetic disorders can easily lead to local recurrence. Unfortunately, the genetic examination, was not available at our department to demonstrate the connection of a malignant transformation of osteoblasts or the possibility of a local recurrence on HA covered implants.

Sailaja G.S. et al demonstrated in an in vitro study that Human osteosarcoma (HOS) cell has an increased affinity for cell anchorage and viability thus proliferation in HA impregnated chitosan. [11] Another recent study by Zhongly S. et al showed that the size of the NanoHA has an inhibition effect for MG 63 and U2OS osteosarcoma cell lines. The study also underlines the importance of cell specific toxicity depending on the Nano HA particle size, damaging mitochondrial activity by increased intracellular $\mathrm{Ca}^{2+}$ levels and caspaze- 9 activity. The exact molecular explication of these properties of HA is not well described; further studies are required to describe the inhibition effect of Nano HA particles on sarcoma cells [12].

We believe that IHC examination with the use of MDM2, p53, HiF-1 $\alpha$ (hypoxia inducible factor) markers can help to understand the risks of local recurrence and chemotherapy resistance of osteosarcoma.

In the presented case the increased Ki67 proliferation index can cover a potential p53 gene mutation or an increased MDM2 protein activity, well known negative prognostic factors for tumor cell invasion, metastases and local recurrence. The recurrence after chemotherapy also underline the aggressiveness of these tumor cells. Based on the literature, a possible explication can be the hypoxic condition in the tumor tissue.In anaerobe condition, cell metabolism lead to a decreased level of ATP, which is crucial for active transmembrane transport of chemotherapy substances, like Doxorubicin, thus drug resistance can occur [15]. Another explication for chemotherapy resistance 
and local recurrence is the mutation of the transmembrane transporter protein (SLC22 A16), crucial for doxorubicin transport [16-19].

The heterogeneity of these sarcomas can be explained by the phenomena of chromothripsis with numerous genetic alterations, mutations, which can occur in any fast dividing cell with damaged cell cycle arrest mechanisms, or repairing molecules like DNA helicases [13].

These possibilities can be examined by the use of the aforementioned IHC markers at the time of biopsy to predict the potential risk of chemotherapy resistance, local recurrence thus further tumor invasion and tumor cell dissemination.

\section{Conclusions}

Local recurrence reveals the increased aggressiveness of a bone sarcoma, with drug-resistance, tumor cell dissemination and possible metastasis formation. The complexity of this problem requires more accurate diagnostic methods to discover the most important prognostic elements, to an early discovery of a potential chemotherapy resistance or local recurrence. The more we know about the histological phenotype of the tumor, the better therapeutic strategy can be applied.

Implants surely influences tumor metabolism, tumor growth. The reduced number of these research papers, requires a higher attention on applied therapy, and more studies to understand the molecular mechanism of tumor growth and drug resistance.

The future is promising with new, personalized therapies, based on the histologic subtype of the tumor.

\section{References}

1.ROZEMAN, L. B. , CLETON-JANSEN, A. M. , HOGENDOORN, P. C. W. , Pathology of primary malignant bone and cartilage tumours, International Orthopaedics (SICOT) (2006) 30, p.437-444

2.ERSTAD,D.J, READY, J., ABRAHAM, J., FERRONE, M.L., BERTAGNOLlI, M.M, BALDINI, E.H, RAUT, C.P., Amputation for extremity sarcoma: contemporary indications and outcomes. Annals of surgical oncology. 2018 Feb 1;25(2),p394-403.

3.MERCADANTE, S.,Malignant bone pain: pathophysiology and treatment. Pain. 1997 Jan;69(1-2),p1-18.

4.HONG, A.M, MILlingTON, S., AHERN, V., MCCOWAGE, G., BOYLE,R., TATTERSALl, M., HAYDU, L., STALLEY, P.D., Limb preservation surgery with extracorporeal irradiation in the management of malignant bone tumor: the oncological outcomes of 101 patients. Annals of Oncology. 2013 Jul 12;24(10),p2676-2680.

5.ENE,R., PANTI, Z.A, NICA, M, POPA, M.G, CIRSTOIU, M.M, MUNTEANU, O., VASILESCU, S.L, SIMION, G., VASILESCU, A., DAVIȚOIU, D.V, CÎRSTOIU, F.C. Chondrosarcoma of the pelvis-case report. Rom J MorpholEmbryol. 2018 Jan 1;59(3),p927-931.

6.EVOLA, F.R, COSTARELLA, L., PAVONE, V, et al. Biomarkers of Osteosarcoma, Chondrosarcoma, and Ewing Sarcoma. Front Pharmacol. 2017;8:150. Published 2017 Apr 7. doi:10.3389/fphar.2017.00150

7.MARDANPOUR, K., MAHTABRAHBAR, S. M., "Coexistence of HER2, Ki67, and p53 in Osteosarcoma: A Strong Prognostic Factor.” North American Journal of Medical Sciences 8.5 (2016),p210-214. PMC. Web. 29 Oct. 2017.

8.RUTH RUBIO et al. - Bone Environment is Essential for Osteosarcoma Development from Transformed Mesenchymal Stem Cells, STEM CELLS 2014;32: p.1136-1148

9.GELLER, D.S, GORLICK, R,. Osteosarcoma: A review of diagnosis, management, and treatment strategies. ClinAdvHematolOncol. 2010; 8,p705-718.

10.CIRSTOIU, C., ENE,RA, PANTI, Z, ENE, P, CIRSTOIU, M., Particularities of Shoulder Recovery After Arthroscopic Bankart Repair with Bioabsorbable and Metallic Suture Anchors. MaterialePlastice. 2015 Sep 1;52(3),p361-3.

11.SAILAJA, G.S., RAMESH, P. , KUMARY, T.V. VARMA, H.K., - Human osteosarcoma cell adhesion behaviour on hydroxyapatite integrated chitosan-poly(acrylic acid) polyelectrolyte complex -ActaBiomaterialia 2; 2006; p 651-657

12.ZHONGLI, SHI, XIN HUANG, B., HUIMIN, T.,Biological Response Of Osteosarcoma Cells To Size-Controlled Nanostructured Hydroxyapatite, Journal Of Biomaterials Applications Volume 25 - July 2010

13.LORENZ S, BARØY T, SUN J, et al. Unscrambling the genomic chaos of osteosarcoma reveals extensive transcript fusion, recurrent rearrangements and frequent novel TP53 aberrations. Oncotarget. 2015;7(5),p5273-5288. doi:10.18632/oncotarget.6567

14.PARK, H.R, JUNG ,W.W, BERTONI, F., BACCHINI, P., PARK ,J.H, KIM Y.W, Park, Y.K.,Molecular analysis of p53, MDM2 and H-ras genes in low-grade central osteosarcoma.Pathol Res Pract. 2004;200(6),p439-45.

15.ZHAO, C., ZHANG, Q., YU, T., SUN, S., WANG, W., LIU, G., Hypoxia promotes drug resistance in osteosarcoma cells via activating AMP-activated protein kinase (AMPK) signaling. J Bone Oncol. 2016;5(1),p22-29.

16.OKABE, M., UNNO,M., HARIGAE, H., KAKU, M., OKITSU, Y., SASAKI ,T., MIZOI, T., SHIIBA, K., TAKANAGA, H., TERASAKI, T., MATSUNO, S., SASAKI I, ITO, S., ABE ,T., Characterization of the organic cation transporter SLC22A16: a doxorubicin importer.BiochemBiophys Res Commun. 2005 Aug 5;333(3),p754-762.

17. FILIP, C.R., UNGUREANU,A., MITARIU,M.C.,"Paniful tonic spasms and brainstem involvement in a patient with neuromyelities optica spectrum disorders"Polish Journal of Neurology and Neurosurgery, vol.50,2016,nr.1,p55-58

18.UNGUREANU,A.,RUS,L., GLIGOR,F.,LAZAROAE,A., PRODAN,L., FILIP,C.R.,"Intravenous levetiracetam as second line treatment for status epilepticus"Farmacia, ,vol 64, nr.4, 2017, p.507-511

19.FILIP,C.R.,UNGUREANU,A, PRAVARIU,I.,"Balo like-lesion associated with psoriazis and autoimmune thyroiditis-case report" Acta NeurologicaBelgica ,vol 115,nr.4 ,2015,p793-796

$\overline{\text { Manuscript received: } 14.10 .2019}$ 